\title{
A Simple Technique to Accomplish Total Muscular Coverage in Complicated Cases of Tissue Expander-Based Breast Reconstruction
}

\author{
Nikolaos V. Michalopoulos ${ }^{1}$ Apostolos S. Mitrousias \\ Ioannis Flessas ${ }^{1}$ George C. Zografos ${ }^{1}$ \\ ${ }^{1}$ Breast Unit, 1st Department of Propaedeutic Surgery, University of \\ Athens, Hippocratio General Hospital, Athens, Greece
}

Indian J Plast Surg 2020;53:152-153

Postmastectomy reconstruction has significant benefits to a woman's body image, self-esteem, and quality of life. ${ }^{1}$ A common way to perform reconstruction is a two-stage procedure starting with the placement of an expander that in a later operation will be replaced with a permanent silicone implant. ${ }^{2}$ Ideally, the expander is inserted in a submuscular pocket that is created by lifting the pectoralis major and serratus anterior muscles. Dissection for creating the pocket extends to the origins of the pectoralis major inferomedially and the serratus anterior and/or its fascia laterally. The two muscles are then sutured together to achieve total muscular coverage of the implant that minimizes the possibility of future complications. Lifting of the pectoralis major muscle may sometimes be difficult due to anatomic variants such as high origin of the muscle, thin muscle, or a surgical injury during dissection. ${ }^{3}$ In these cases, a muscular tear may occur that more often happens at the inferior attachment of the pectoralis major to the cartilage of the second to sixth rib or to the anterior rectus sheath ( $\mathbf{- F i g .} \mathbf{1}$ ). This leaves the expander uncovered and prone to later infection. The defect is often difficult to repair as the muscle contracts and cannot be easily reapproximated with sutures due to the existing tension.

We describe a technique that can overcome such difficulties and provide total muscular coverage of the expander in cases of small areas of exposed expander/ implant. Subcutaneous tissue arising from anterior surface of the distal attachment of the rectus abdominal muscle to the costal cartilages of ribs five to seven can be mobilized, and, essentially being a turn over flap, it can be placed over the defect, and fixed to the pectoralis major muscle with absorbable Vicryl 3-0 sutures (-Figs. 2 and 3 ). The flap's blood supply arises mainly from branches of the superior epigastric artery. Care should be taken not to perform extensive mobilization of this advancement flap

\section{Vasileios Kalles ${ }^{1}$ Constantinos G. Zografos ${ }^{1}$}

Address for correspondence Vasileios Kalles, MD, MSc, PhD, Hippocratio General Hospital, 114 Vas. Sofias Avenue, GR-11527 Athens, Greece (e-mail: vassilis_kalles@yahoo.gr).

to avoid potential necrosis due to compromised blood supply. The flap is mobilized along with 3 to $5 \mathrm{~mm}$ of overlying fat. Mobilization of thicker fat pad could create aesthetic problems (bulging), compromise the inferior pole skin blood supply, or, in case of fat necrosis, differential diagnostic problems on imaging studies as its imaging features might resemble those of breast cancer recurrence. Thereafter, the procedure is continued as normal. With this simple procedure, the expander is fully covered, as an interstitial layer between the expander and the mastectomy flap exists, which provides increased protection and minimizes the risk of expander infection and extrusion

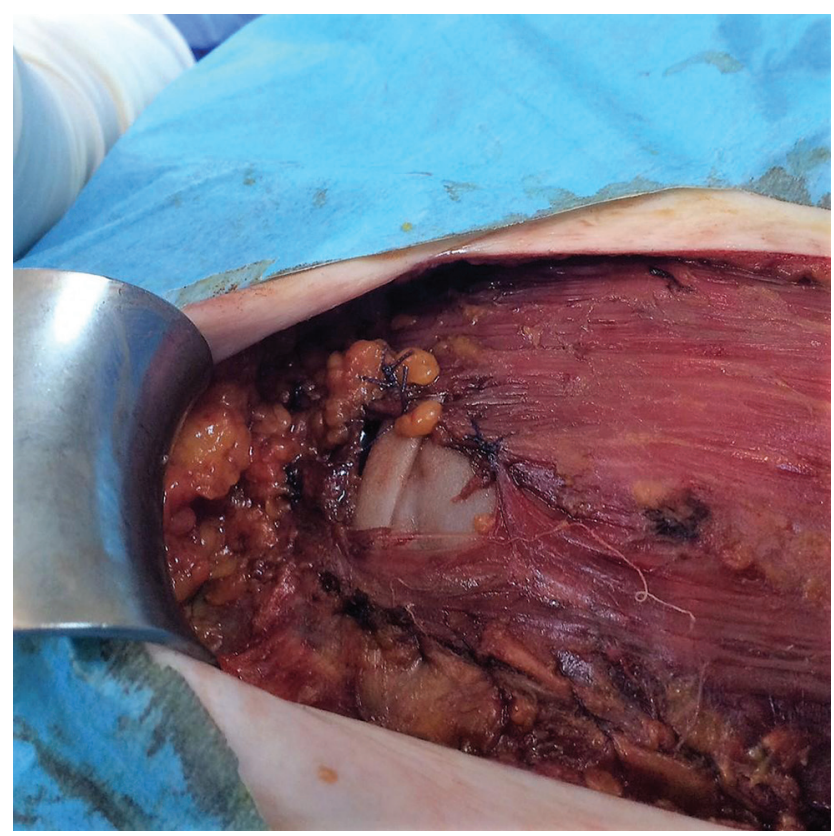

Fig. 1 Pectoralis major muscular tear leaving expander's anterior surface exposed.
DOI https://doi.org/

$10.1055 / \mathrm{s}-0039-3402355$

ISSN 0970-0358.
License terms

(요 (1) $\Theta \circledast$ 


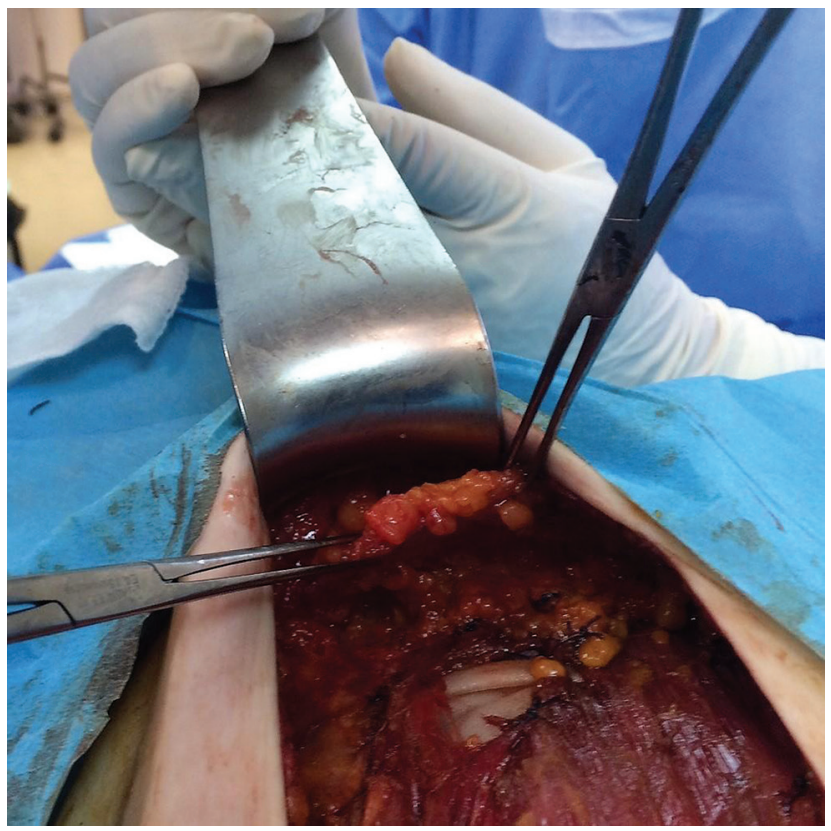

Fig. 2 Advancement flap from the anterior surface of the rectus sheath mobilized.

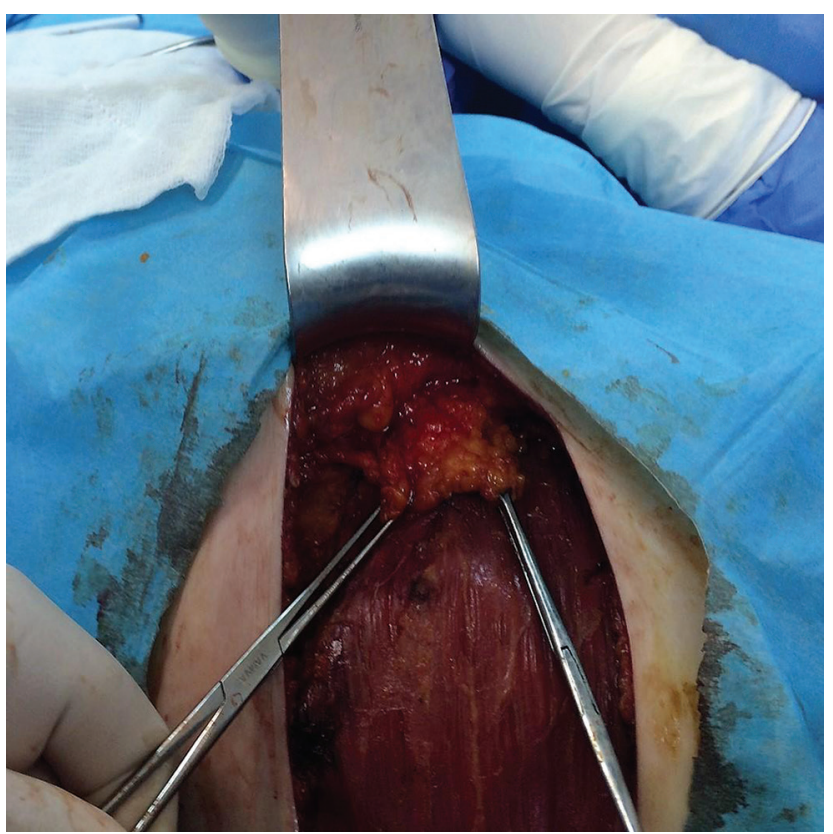

Fig. 3 Flap fixation with absorbable sutures over the muscular defect.

( - Fig. 4). Postoperatively, the vascularity of the flap is not compromised once the tissue expander is expanded in small steps.

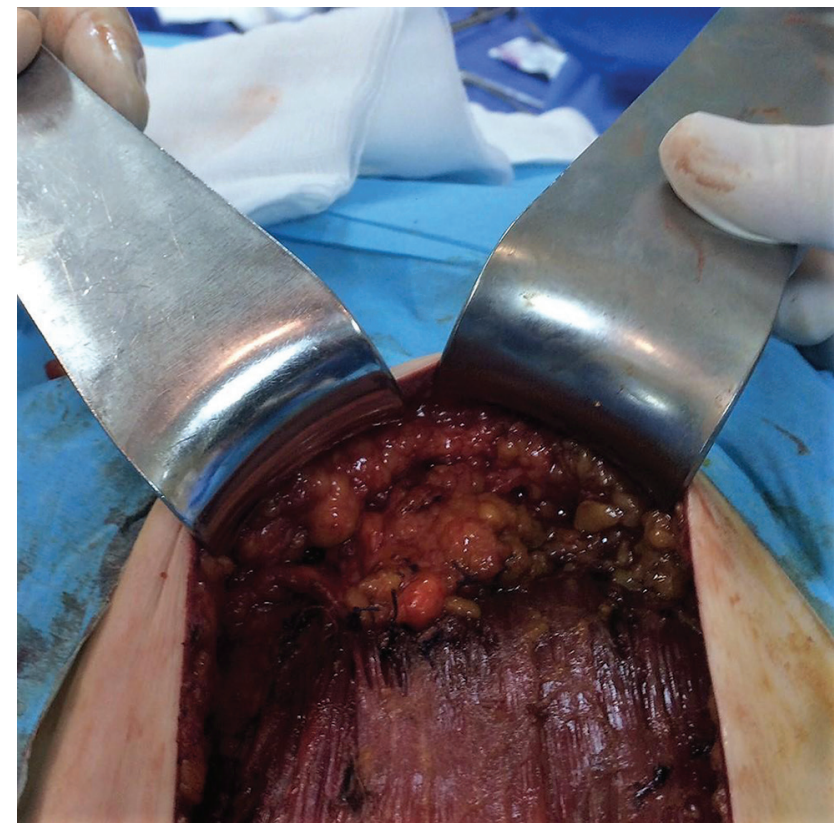

Fig. 4 Final result with total coverage of the expander.

Compared with other available strategies of tissue expander coverage, such as rectus sheath turnover flap or the use of biological mesh, this technique represents a simple and cost-effective approach, avoiding an extended dissection in the area or the significant cost of a prosthetic mesh; however, it is applicable in small defects, and other techniques should be considered if adequate coverage of the defect is not feasible.

The technique offers a simple and easy-to-perform solution in case of "pocket" defects occurring during expander placement as a first stage of breast reconstruction following mastectomy.

\section{Acknowledgment}

This article received no specific grant from any funding agency in the public, commercial, or not-for-profit sectors.

\section{References}

1 Howard-McNatt MM. Patients opting for breast reconstruction following mastectomy: an analysis of uptake rates and benefit. Breast Cancer (Dove Med Press) 2013;5(5):9-15

2 Serletti JM, Fosnot J, Nelson JA, Disa JJ, Bucky LP. Breast reconstruction after breast cancer. Plast Reconstr Surg 2011;127(6):124e-135e

3 Huber KM, Boyd TG, Quillo AR, Wilhelmi BJ. Implications of anomalous pectoralis muscle in reconstructive breast surgery: the oblique pectoralis anterior. Eplasty 2012;12:e44 